
\title{
25 Research Soure \\ Butterfly Glioblastoma - the Impact of Tumor Volumes and Treatment Strategies on Clinical Outcome
}

\section{Line Sagerup Bjorland ( $\square$ line.bjorland@sus.no )}

Stavanger University Hospital: Department of Oncology https://orcid.org/0000-0003-3043-7804

Kathinka Dæhli Kurz

Stavanger University Hospital: Department of Radiology

\section{Fluge Øystein Fluge}

Haukeland University Hospital: Department of Oncology and Medical Physics

Bjørnar Gilje

Stavanger University Hospital: Department of Oncology

\section{Rupavathana Mahesparan}

Haukeland University Hospital: Department of Neurosurgery

\section{Hege Sætran}

Haukeland University Hospital: Department of Pathology

\section{Anastasia Ushakova}

Stavanger University Hospital: Department of Research, Section of Biostatistics

\section{Elisabeth Farbu}

Stavanger University Hospital: Department of Neurology

\section{Research Article}

Keywords: Butterfly glioblastoma, bihemispheric glioma, corpus callosum, 3D volumetric, survival, clinical outcomes

Posted Date: December 10th, 2021

DOI: https://doi.org/10.21203/rs.3.rs-1010346/v2

License: (c) (i) This work is licensed under a Creative Commons Attribution 4.0 International License. Read Full License 


\section{Abstract}

\section{Purpose}

Butterfly glioblastoma is a rare subgroup of glioblastoma with a bihemispheric tumor crossing the corpus callosum, and is associated with a dismal prognosis. Prognostic factors are previously sparsely described and optimal treatment approaches remain uncertain. We aimed to analyse prognostic factors in butterfly glioblastoma, and to evaluate treatment strategies and outcome in a real-world setting.

\section{Methods}

We conducted a retrospective population-based cohort study of patients diagnosed with butterfly glioblastoma in Western Norway between 01/01/2007 and 31/12/2014. Clinical data were extracted from electronic medical records. Molecular and MRI volumetric analyses were retrospectively performed. Survival analyses were performed using Kaplan-Meier method and Cox proportional hazards regression models.

\section{Results}

Among 381 patients diagnosed with glioblastoma, 33 patients $(8.7 \%)$ met the criteria for butterfly glioblastoma. Median overall survival was 5.5 months $(95 \% \mathrm{Cl} 3.1-7.9)$ and three-year survival was $9.1 \%$. Older age and mainly deep-seated tumour location were associated with poor outcome, with adjusted hazard ratio (HR) 1.06 (95\% Cl 1.03-1.10), p<0.001, and adjusted HR 4.58 (95\% Cl 1.15-18.20), $\mathrm{p}=0.03$. Best supportive care was associated with poorer survival compared to multimodal treatment (adjusted HR 5.11 (95\% Cl 1.09-23.89), $p=0.04)$.

\section{Conclusion}

Outcome from butterfly glioblastoma was dismal, with a median overall survival of less than six months. However, long-term survival was comparable to that observed in glioblastoma in general, and multimodal treatment was associated with longer survival. This suggests that patients with butterfly glioblastoma may benefit from a more comprehensive treatment approach despite the overall poor prognosis.

\section{Introduction}

Butterfly glioblastoma is a subgroup of glioblastoma where tumor crosses the corpus callosum and affects both hemispheres [1, 2]. Previous studies have reported diverging incidence rates, ranging from 2.2 to $14.3 \%$ of adults diagnosed with glioblastoma [3-5]. It has been suggested that butterfly glioblastoma is clinically distinct from non-butterfly glioblastoma, being associated with less aggressive treatment, larger tumor volumes, and a poorer outcome [3]. It is uncertain whether the poorer prognosis is a consequence of intrinsic tumor features or due to the choice of treatment strategies. Improved knowledge on prognostic factors and treatment, and their impact on outcome, is required to enhance clinical decision-making. Few previous publications have described the treatment and outcome of 
butterfly glioblastoma [3-8]. The optimal treatment approach remains an unresolved concern, as it is uncertain if results from studies on glioblastoma in general are applicable to this subgroup. A few studies have suggested a benefit from an aggressive treatment approach despite the poor prognosis $[3,4,8]$. There is no definitive consensus on recurrent glioblastoma treatment [9]. To the best of our knowledge, treatment of recurrent butterfly glioblastoma has not been described previously.

Isocitrate Dehydrogenase (IDH) mutations are positive prognostic factors in glioma, but according to the 2021 WHO classification of tumors of the central nervous system, IDH mutated astrocytoma will no longer be classified as glioblastoma [10, 11]. O(6)-methylguanine-DNA methyltransferase (MGMT) promoter methylation is a favourable prognostic factor in glioblastoma [12]. BRAF mutation is uncommon in glioma, but is regularly seen in epithelioid glioblastoma [13]. Molecular characteristics of butterfly glioblastoma have only been described in small subsets in a few studies, and the prognostic value in this subgroup is not clarified [4, 8]. Due to the deep-seated tumor location of butterfly glioblastoma, MRI may serve as a useful non-invasive prognostic tool and help to determine the preferred treatment strategy. A few previous studies have described MRI volumetric analyses in butterfly glioblastoma [3-6].

We aimed to evaluate the prognostic values of clinical, molecular, and radiological characteristics, including volumetric analyses of T1-weighted and T2-weighted and/or fluid attenuated inversion recovery (FLAIR) MRI, and to identify real-world treatment strategies and outcome from butterfly glioblastoma.

\section{Methods}

\section{Design and sample}

We conducted a population-based, retrospective cohort study with a follow-up of seven years, or until death. We identified all patients diagnosed with glioblastoma in Rogaland and Vestland counties of Western Norway, including ten secondary and tertiary referral centres, between 01/01/2007 and $31 / 12 / 2014$. They included patients with histologically confirmed glioblastoma and those where the diagnosis was based on typical MRI pattern only, and where differential diagnoses were considered highly unlikely. We defined butterfly tumor as a contrast-enhancing tumor crossing the corpus callosum and affecting both hemispheres, corresponding to comparable studies $[3,4,8]$. Patients diagnosed with glioblastoma and with an MRI pattern meeting the butterfly tumor criteria were eligible for inclusion.

\section{Measures}


Patient characteristics and clinical findings were extracted from electronic health records (table 1). In cases where it was not documented, Karnofsky Performance Status (KPS) was retrospectively determined based on information in health records. Comorbidity burden was retrospectively assessed using Charlson comorbidity score [14].

Histological samples were re-evaluated by a neuropathologist. Molecular analyses were retrospectively performed on primary tissue samples. O(6)-methylguanine-DNA methyltransferase (MGMT) promoter methylation was analysed using methylation-specific polymerase chain reaction (MSP) as a qualitative method [15]. Determination of IDH mutational status was restricted to identification of the most frequent mutation, IDH1 R132H, and detected by immunohistochemistry with an IDH1 R132H specific monoclonal antibody. Identification of BRAF mutational status was restricted to mutation of codon 600 of exon 15 (V600E), being the most frequent activating mutation of the BRAF gene. Sanger DNA sequencing of exon 15 , sequencing both the forward and the reverse strand, was performed.

MR images at time of diagnosis were re-evaluated by a neuroradiologist. Images were acquired from four different hospitals over an eight-year period, and imaging protocols varied regarding sequences and quality. T1-weighted series prior to, and after, intravenous gadolinium containing contrast agent, and T2weighted and/or FLAIR series were present in all patients. Tumor characteristics were identified (table 1). Main location was defined as the lobe/region mainly affected by tumor. Involvement of the corpus callosum was classified according to Highley and colleagues [16]. Mass effect was measured as millimetres ( $\mathrm{mm}$ ) midline shift, and was classified as slight or severe with a cut off value of $10 \mathrm{~mm}$, in line with a comparable study [17]. Contrast-enhancing tumor on T1-weighted series and tumor associated non-enhancing hyperintense lesions on T2-weighted/FLAIR series were identified, according to standardized neuro-oncological tumor assessment [18]. Volumes were delineated using the open source software platform 3D slicer Version 4.10.1, and measured in cubic centimetres $\left(\mathrm{cm}^{3}\right)$. Necrosis was included in the T1 volumes, and T1 volumes were included in the T2/FLAIR volumes.

Treatment and complications in primary and recurrence situations were registered (table 2). As discontinuation of, or changes in, treatment is a frequent concern, the completion of radiation therapy (60 Gy in 2 Gy fractions) with concurrent Temozolomide (TMZ) administered daily during the entire period, and the completion of at least one of six planned TMZ monotherapy courses, was classified as treatment according to the Stupp protocol [19]. All other combinations of radiation therapy and/or TMZ were classified as less-intensive chemoradiotherapy. Time of diagnosis was defined as the date of the first MRI, and progressive disease as unequivocal clinical or radiological progression. Long-term survival was defined as survival of three years or more, in line with previous studies $[20,21]$. 


\section{Statistics}

Statistical analyses were performed using Statistical Package for the Social Sciences (SPSS) Version 16, and figures were created in R Version 4.0.4. Categorical data were presented as counts and percentages, and the between-group differences were assessed using Chi square $\left(\chi^{2}\right)$ and Fisher's exact test, as appropriate. Continuous variables were presented as mean \pm standard deviation (SD) or median and range or interquartile range (IQR). Comparison of two and multiple groups were performed using MannWhitney $\mathrm{U}$ and Kruskal-Wallis test, respectively. Correlations between continuous variables were assessed using Pearson correlation test, and presented as Pearson correlation coefficient ( $r$ ). Survival analyses were performed using Kaplan-Meier plots and Cox proportional hazards regression models. To investigate the relations between patient characteristics and survival, we applied an unadjusted Cox model and a Cox model adjusted for age and sex. No correction for multiple comparison was made. $P$ values $<0.05$ were considered statistically significant.

\section{Results}

We identified 381 patients diagnosed with glioblastoma in the study period. This cohort was described in a previous study [22]. Forty patients had a bihemispheric tumor crossing the corpus callosum. Three patients diagnosed by computed tomography (CT) only and one not meeting the histopathological criteria for glioblastoma were excluded. IDH1 mutation was detected in three patients and these were excluded. Finally, 33 patients ( $8.7 \%$ of patients diagnosed with glioblastoma) met the criteria for butterfly glioblastoma and were enrolled. Median age was 66.6 years (range 27.9-84.8) and median Karnofsky Performance Status was 80 (range 20-100). Patient characteristics are listed in table 1.

Glioblastoma was histologically confirmed in 13 patients (39.4\%), for the remaining 20 patients (60.6\%) the diagnosis was based on MRI pattern only. The latter group included two patients with nonrepresentative biopsies. Among the 13 patients with conclusive biopsies, 11 samples were available for further analyses. Radiological, histopathological, and molecular characteristics are presented in table 1. Sixteen patients (48.5\%) presented with a midline shift, mean $7.9 \mathrm{~mm}(\mathrm{SD} \pm 3.3)$, and with a severe midline shift of $10 \mathrm{~mm}$ or more in four patients.

(Table 1 approximately here)

Median contrast-enhancing tumor volume in T1-weighted images was $41 \mathrm{~cm}^{3}$ (range 2-146). Median volume of non-enhancing hyperintense lesions in T2-weighted/FLAIR images was $137 \mathrm{~cm}^{3}$ (range 25- 
340). The ratio between T2/FLAIR and T1 volumes ranged from 1.1 to 12.5 . Tumor volumes and locations are shown in figure 1. Median T1 volumes were smallest in central and occipital tumors with median volumes of $37 \mathrm{~cm} 3$ (IQR 8-46) and $28 \mathrm{~cm} 3$ (IQR 22-48), however without statistical difference $(p=0.07)$. There was no difference in median T2/FLAIR $(p=0.11)$ or T2/T1 ratio $(p=0.10)$ between different locations.

(Figure 1 approximately here)

Both median T1 volume and median abnormal T2/FLAIR volume were larger in the resection group than the non-resection group: $109 \mathrm{~cm}^{3}$ vs $39 \mathrm{~cm}^{3}(p=0.005)$ and $281 \mathrm{~cm}^{3}$ vs $109 \mathrm{~cm}^{3}(p=0.02)$. Larger T1 volume was correlated with younger age $(r=-0.365(p=0.04))$, while no correlation between T2 volume and age was observed $(r=-0.072(p=0.69))$. All three patients with the largest T1 volumes $\left(\geq 100 \mathrm{~cm}^{3}\right)$, and three of four patients with the largest T2 lesions $\left(\geq 300 \mathrm{~cm}^{3}\right)$, were aged under 70 years.

\section{Treatment and survival}

Treatment and complications are outlined in table 2. Among patients aged under 70 years, 19 of 20 $(95.0 \%)$ received radiation therapy, compared to eight of 13 patients $(61.5 \%)$ aged over 70 years $(p=0.03)$. Among patients aged under 70 years, 18 of $20(90.0 \%)$ received multimodal treatment, compared to two of 13 patients $(15.4 \%)$ aged over 70 years $(p<0.0001)$. Hypofractionated radiation therapy was planned in four of six patients treated with best supportive care, but cancelled because of a rapid clinical deterioration.

(Table 2 approximately here)

Median overall survival was 5.5 months ( $95 \% \mathrm{Cl} 3.1-7.9)$, and median progression-free survival 3.8 months (95\% $\mathrm{Cl} 3.0-4.7)$. One-, two-, and three-year survival rates were $15.2 \%, 12.1 \%$, and $9.1 \%$. Median survival in patients treated according to the Stupp protocol was 8.0 months ( $95 \% \mathrm{Cl} 7.0-9.0)$, compared to 5.5 months $(95 \% \mathrm{Cl} 1.9-9.1)$ and 1.6 months $(95 \% \mathrm{Cl} 0.5-2.6)$ in patients treated with less-intensive chemoradiotherapy and best supportive care $(p<0.001)$. Median survival in patients aged over 70 years was 2.1 months ( $95 \% \mathrm{Cl} 0.0-5.0$ ), compared to 7.1 months ( $95 \% \mathrm{Cl}$ 5.6-8.6 months) in patients aged under 70 years $(p=0.001)$. Nine patients $(27.3 \%)$ died within three months of diagnosis. Overall survival in 13 
patients with histologically confirmed butterfly glioblastoma was 8.0 months (95\% $\mathrm{Cl} 4.9-11.0)$. Survival curves are presented in figure 2 .

(Figure 2 approximately here)

Older age and mainly deep-seated tumor location was associated with poor survival according to Cox regression adjusted for age, sex, and KPS, with HR $1.06(p=0.003)$ and HR $4.58(p=0.03)$, respectively. There was not revealed any associations between T1 or T2/FLAIR volumes and outcome. Best supportive care was associated with poorer outcome compared to multimodal treatment (HR 5.11, p=0.04), whereas the impact from one treatment modality was not significant (HR 1.67, $p=0.46)$. Unadjusted and adjusted analyses are presented in table 3 .

(Table 3 approximately here)

All three long-term surviving patients were females aged under 70 years with good performance scores (KPS $\geq 90$ ), and two of them had a histologically confirmed IDH1 wildtype glioblastoma. These two had both a severe midline shift, although highly different tumor volumes, with T1 volume, T2/FLAIR volume, and T2/FLAIR:T1 ratio of $11 \mathrm{~cm}^{3}$ vs $140 \mathrm{~cm}^{3}, 108 \mathrm{~cm}^{3}$ vs $255 \mathrm{~cm}^{3}$, and $9.8 \mathrm{vs} 1.8$, respectively.

Two patients (6.1\%) had stable disease with no sign of recurrence during follow-up. Thirteen patients (39.4\%) had no period of improvement or stable disease after primary diagnosis, presenting with a continuous progression and deterioration until death, without being diagnosed with recurrence. In the remaining 18 patients (54.5\%), an interval with improvement or stabilisation after primary treatment was observed, thereafter being diagnosed with recurrence. Seven of 18 patients (38.9\%) diagnosed with recurrence received various anti-tumor treatments (table 2). Median post-recurrence survival was 4.1 months $(95 \% \mathrm{Cl} 2.8-5.4)$ in patients receiving anti-tumor treatment, compared to 1.5 month $(95 \% \mathrm{Cl} 0.9$ $2.1)$ in patients receiving best supportive care $(p=0.04)$.

\section{Discussion}

In this retrospective population-based cohort study we found that $8.7 \%$ of adults diagnosed with glioblastoma met the criteria for butterfly glioblastoma. Outcome was poor, with a median overall survival of less than six months. This study adds real-world data on prognostic factors and outcome in a 
previously sparsely described subgroup of glioblastoma, whereas previous studies are based on data from tertiary referral centres $[3-5,8]$.

\section{Survival}

Our study confirms previous findings of a considerably poorer prognosis in butterfly glioblastoma compared to glioblastoma in general, where population-based studies observed a median overall survival of nine to 11 months [23-26]. A median overall survival of 5.5 months in our cohort was comparable to the results from previous butterfly glioblastoma studies, with median survival ranging from 3.2 to 5.9 months $[4,5,8]$. Survival was substantially poorer than that observed by Burks and colleagues, where median survival was 12 and 15 months in patients who underwent two different surgical approaches [6]. Likely explanations are different selection criteria and settings, and highly different resection rates of $12 \%$ and $100 \%$. Although these two studies cannot be compared directly, this may indicate a potential benefit from a more comprehensive treatment approach in patients with butterfly glioblastoma. Patients aged over 70 years received less intensive treatment than younger patients, and had a significantly poorer outcome with a median survival of only two months, equal to the findings of Dayani and colleagues [4]. Previous studies have demonstrated that combined chemoradiotherapy improves outcome in elderly glioblastoma patients with adequate performance status [27-29]. Future studies, preferably including quality of life analyses, may clarify if these results are applicable to the butterfly glioblastoma subgroup.

Despite the poor overall outcome, patients with long-term survival was observed in $9.1 \%$ of the patients, not markedly poorer than pooled three-year survival rate of $11 \%$ in a large meta-analysis of glioblastoma in general [30]. We suggest that patients with butterfly glioblastoma might receive less-intensive treatment due to the deep-seated tumor location, uncertainty of treatment benefit, and the dismal prognosis. This study demonstrates that long-term survival is possible, supporting an argument for a higher treatment intensity in patients in acceptable general condition. A second argument for providing more multimodal treatment is the challenge in predicting treatment benefit, long-term survival, and quality of life in these patients. Only a minority of patients receiving TMZ suffered from significant toxicity, serving as a third argument to promote an increased treatment intensity in patients with butterfly glioblastoma. Nearly $40 \%$ of the patients diagnosed with recurrent glioblastoma received anti-tumor treatment, and had a median post-recurrence survival of approximately four months. We suggest that anti-tumor treatment may be appropriate in patients with recurrent butterfly glioblastoma and acceptable performance status.

\section{MR volumetric analyses}


All three patients with the largest tumor volumes in T1-weighted MRI, and three of four patients with the largest abnormal T2/FLAIR volumes, were aged under 70 years. Among possible explanations, we suggest that younger patients, having a higher cognitive reserve, tolerate larger tumor growth before symptoms occur. Further, the tumor volumes of long-term surviving patients differed widely, ranging from the upper to lower quartiles. We suggest that larger tumor volumes may not be considered an unequivocal negative prognostic factor, as larger volumes may be seen in younger patients harbouring favourable prognostic factors. The association between T2/FLAIR volumes and age has not been studied previously, thus comparison with other studies was not applicable.

In our cohort, both median T1 and T2/FLAIR volumes were larger in the resection group than the nonresection group. Larger tumor volumes in younger patients and larger tumor volumes in frontal lobes, with favourable surgical accessibility, are possible explanations. In contrast, Dziurzynski and colleagues reported slightly larger, but statistically insignificant, median T1 volume in the non-resection group compared to the resection group, although ranges were wide in both groups and with no difference in FLAIR volumes [5]. Among possible explanations for these differences are the different settings, small sample sizes, low resection rates of $16 \%$ and $36 \%$, and the lack of adjustment for molecular subgroups. Unlike Dayani and colleagues, who reported a slight negative correlation between larger T1 volume and survival, we found no such association [4]. Small sample sizes and wide range of tumor volumes may explain this uncertainty. This study did not reveal any significant associations between T1 or T2/FLAIR volumes and survival, however this may not be evaluable in this small sample.

\section{Strengths and limitations}

Strengths of this study included the population-based study design and that all patients were followed up for seven years or until death. Furthermore, clinical information on all patients was available in a common electronic record, and T1-weighted and T2-weighted/FLAIR images were available in all patients. The small sample was the main limitation, related to the rarity of the condition, but is comparable to sample sizes in previous studies $[3-5,8]$. Further, the lack of histological samples in approximately $60 \%$ of the enrolled patients was a significant limitation, causing a risk of inclusion bias, and the high number of missing molecular data. Also, associations between treatment and outcome in elderly patients were inconclusive, as only a minority of elderly patients received multimodal treatment.

Outcome from butterfly glioblastoma was dismal, with a median overall survival of less than six months. However, long-term survival was comparable to that observed in glioblastoma in general, and multimodal treatment was associated with longer survival. This suggests that patients with butterfly glioblastoma may benefit from a more comprehensive treatment approach despite the overall poor prognosis. 


\section{Declarations}

\section{Conflicts of Interest/Competing Interests}

The authors have no relevant financial or non-financial interests to disclose.

\section{Funding}

This study received grants from Family Blix Foundation and Svanhild and Arne Must Foundation. The Foundations had no influence on protocol, analyses, presentation of results, or any other part of the project.

\section{Acknowledgments}

The authors would like to thank Joanna Haynes for proofreading the article.

\section{Author contributions}

All authors contributed to the study conception and design. Material preparation and data collection were performed by Line Sagerup Bjorland, Kathinka Dæhli Kurz and Hege Sætran. MRI analyses were performed by Kathinka Dæhli Kurz. Hege Sætran was responsible for histopathological and molecular analyses. Statistical analyses were performed by Line Sagerup Bjorland and Anastasia Ushakova. All authors participated in the interpretation of the results. The first draft of the manuscript was written by Line Bjorland, and all authors commented on previous versions of the manuscript. All authors read and approved the final manuscript.

\section{Compliance with Ethical Standard}

The study was approved by the Regional Committees for Medical and Health Research Ethics (REK Vest 2014/1931). Informed consent was obtained from patients alive at time of inclusion. Waiver of consent was approved for deceased patients.

\section{Data availability}

The data are not available due to privacy and ethical restrictions, as participants of this study did not agree for their data to be shared publicly. 


\section{References}

1. Bourekas EC, Varakis K, Bruns D, Christoforidis GA, Baujan M, Slone HW, Kehagias D (2002) Lesions of the corpus callosum: MR imaging and differential considerations in adults and children. AJR American journal of roentgenology 179: 251-257 doi:10.2214/ajr.179.1.1790251

2. Ho ML, Moonis G, Ginat DT, Eisenberg RL (2013) Lesions of the corpus callosum. AJR American journal of roentgenology 200: W1-16 doi:10.2214/ajr.11.8080

3. Chaichana KL, Jusue-Torres I, Lemos AM, Gokaslan A, Cabrera-Aldana EE, Ashary A, Olivi A, Quinones-Hinojosa A (2014) The butterfly effect on glioblastoma: is volumetric extent of resection more effective than biopsy for these tumors? Journal of neuro-oncology 120: 625-634 doi:10.1007/s11060014-1597-9

4. Dayani F, Young JS, Bonte A, Chang EF, Theodosopoulos P, McDermott MW, Berger MS, Aghi MK (2018) Safety and outcomes of resection of butterfly glioblastoma. 44: E4 doi:10.3171/2018.3.Focus1857

5. Dziurzynski K, Blas-Boria D, Suki D, Cahill DP, Prabhu SS, Puduvalli V, Levine N (2012) Butterfly glioblastomas: a retrospective review and qualitative assessment of outcomes. Journal of neurooncology 109: 555-563 doi:10.1007/s11060-012-0926-0

6. Burks JD, Bonney PA, Conner AK, Glenn CA, Briggs RG, Battiste JD, McCoy T, O'Donoghue DL, Wu DH, Sughrue ME (2016) A method for safely resecting anterior butterfly gliomas: the surgical anatomy of the default mode network and the relevance of its preservation. 126: 1795 doi:10.3171/2016.5.Jns153006

7. Hall BJ, Maleyko I, Brodbelt A, Jenkinson MD, Chavredakis E (2019) The utility of surgery in butterfly glioblastoma: A case-control study. Journal of Clinical Oncology 37: e13529-e13529 doi:10.1200/JC0.2019.37.15_suppl.e13529

8. Opoku-Darko M, Amuah JE, Kelly JJP (2018) Surgical Resection of Anterior and Posterior Butterfly Glioblastoma. World neurosurgery 110: e612-e620 doi:https://doi.org/10.1016/j.wneu.2017.11.059

9. Weller M, Bent M, Preusser M, Le Rhun E, Tonn J, Minniti G, Bendszus M, Balana C, Chinot O, Dirven L, French P, Hegi M, Jakola AS, Platten M, Roth P, Rudà R, Short S, Smits M, Taphoorn M, Deimling A, Westphal M, Soffietti R, Reifenberger G, Wick W (2020) EANO guidelines on the diagnosis and treatment of diffuse gliomas of adulthood. Nature reviews Clinical oncology 18: 170-186 doi:10.1038/s41571-020-00447-z

10. Sanson M, Marie Y, Paris S, Idbaih A, Laffaire J, Ducray F, Hallani SE, Boisselier B, Mokhtari K, Hoang-Xuan K, Delattre JY (2009) Isocitrate dehydrogenase 1 codon 132 mutation is an important 
prognostic biomarker in gliomas. Journal of Clinical Oncology 27: 4150-4154 doi:10.1200/JC0.2009.21.9832

11. Louis DN, Perry A, Wesseling P, Brat DJ, Cree IA, Figarella-Branger D, Hawkins C, Ng HK, Pfister SM, Reifenberger G, Soffietti R, von Deimling A, Ellison DW (2021) The 2021 WHO Classification of Tumors of the Central Nervous System: a summary. Neuro-oncology 23: 1231-1251 doi:10.1093/neuonc/noab106 \%J Neuro-Oncology

12. Olson RA, Brastianos PK, Palma DA (2011) Prognostic and predictive value of epigenetic silencing of MGMT in patients with high grade gliomas: a systematic review and meta-analysis. Journal of neuro-oncology 105: 325-335 doi:10.1007/s11060-011-0594-5

13. Burger MC, Ronellenfitsch MW, Lorenz NI, Wagner M, Voss M, Capper D, Tzaridis T, Herrlinger U, Steinbach JP, Stoffels G, Langen KJ, Brandts C, Senft C, Harter PN, Bahr O (2017) Dabrafenib in patients with recurrent, BRAF V600E mutated malignant glioma and leptomeningeal disease. Oncology reports 38 : 3291-3296 doi:10.3892/or.2017.6013

14. Charlson M, Szatrowski TP, Peterson J, Gold J (1994) Validation of a combined comorbidity index. Journal of clinical epidemiology 47: 1245-1251 doi:10.1016/0895-4356(94)90129-5

15. Christians A, Hartmann C, Benner A, Meyer J, von Deimling A, Weller M, Wick W, Weiler M (2012) Prognostic value of three different methods of MGMT promoter methylation analysis in a prospective trial on newly diagnosed glioblastoma. PloS one 7: e33449-e33449 doi:10.1371/journal.pone.0033449

16. Highley JR, Esiri MM, McDonald B, Cortina-Borja M, Herron BM, Crow TJ (1999) The size and fibre composition of the corpus callosum with respect to gender and schizophrenia: a post-mortem study. Brain 122: 99-110 doi:10.1093/brain/122.1.99 \%J Brain

17. Wach J, Hamed M, Schuss P, Güresir E, Herrlinger U, Vatter H, Schneider M (2020) Impact of initial midline shift in glioblastoma on survival. Neurosurgical review doi:10.1007/s10143-020-01328-w

18. Wen PY, Macdonald DR, Reardon DA, Cloughesy TF, Sorensen AG, Galanis E, Degroot J, Wick W, Gilbert MR, Lassman AB, Tsien C, Mikkelsen T, Wong ET, Chamberlain MC, Stupp R, Lamborn KR, Vogelbaum MA, van den Bent MJ, Chang SM (2010) Updated response assessment criteria for high-grade gliomas: response assessment in neuro-oncology working group. Journal of clinical oncology : official journal of the American Society of Clinical Oncology 28: 1963-1972 doi:10.1200/jco.2009.26.3541

19. Stupp R, Mason WP, van den Bent MJ, Weller M, Fisher B, Taphoorn MJB, Belanger K, Brandes AA, Marosi C, Bogdahn U, Curschmann J, Janzer RC, Ludwin SK, Gorlia T, Allgeier A, Lacombe D, Cairncross JG, Eisenhauer E, Mirimanoff RO (2005) Radiotherapy plus Concomitant and Adjuvant Temozolomide for Glioblastoma. New England Journal of Medicine 352: 987-996 doi:doi:10.1056/NEJMoa043330 
20. Krex D, Klink B, Hartmann C, von Deimling A, Pietsch T, Simon M, Sabel M, Steinbach JP, Heese O, Reifenberger G, Weller M, Schackert G (2007) Long-term survival with glioblastoma multiforme. Brain 130: 2596-2606 doi:10.1093/brain/awm204

21. Hartmann C, Hentschel B, Simon M, Westphal M, Schackert G, Tonn JC, Loeffler M, Reifenberger G, Pietsch T, von Deimling A, Weller M (2013) Long-term survival in primary glioblastoma with versus without isocitrate dehydrogenase mutations. Clinical cancer research : an official journal of the American Association for Cancer Research 19: 5146-5157 doi:10.1158/1078-0432.Ccr-13-0017

22. Bjorland LS, Fluge O, Gilje B, Mahesparan R, Farbu E (2021) Treatment approach and survival from glioblastoma: results from a population-based retrospective cohort study from Western Norway. BMJ open 11: e043208 doi:10.1136/bmjopen-2020-043208

23. Bruhn H, Strandéus M, Milos P, Hallbeck M, Vrethem M, Lind J (2018) Improved survival of Swedish glioblastoma patients treated according to Stupp. 138: 332-337 doi:10.1111/ane.12966

24. Hansen S, Rasmussen BK, Laursen RJ, Kosteljanetz M, Schultz H, Norgard BM, Guldberg R, Gradel KO (2018) Treatment and survival of glioblastoma patients in Denmark: The Danish NeuroOncology Registry 2009-2014. Journal of neuro-oncology doi:10.1007/s11060-018-2892-7

25. Lwin Z, MacFadden D, AL-Zahrani A, Atenafu E, Miller B, Menard C, Laperriere N, Mason WP (2011) A population-based study of glioblastoma multiforme (GBM) in the new stupp paradigm: Have we improved outcome? Journal of Clinical Oncology 29: 2012-2012 doi:10.1200/jco.2011.29.15_suppl.2012

26. Ronning PA, Helseth E, Meling TR, Johannesen TB (2012) A population-based study on the effect of temozolomide in the treatment of glioblastoma multiforme. Neuro-oncology 14: 1178-1184 doi:10.1093/neuonc/nos153

27. Rusthoven CG, Koshy M, Sher DJ, Ney DE, Gaspar LE, Jones BL, Karam SD, Amini A, Ormond DR, Youssef AS, Kavanagh BD (2016) Combined-Modality Therapy With Radiation and Chemotherapy for Elderly Patients With Glioblastoma in the Temozolomide Era: A National Cancer Database Analysis. JAMA neurology 73: 821-828 doi:10.1001/jamaneurol.2016.0839 \%J JAMA Neurology

28. Perry JR, Laperriere N, O'Callaghan CJ, Brandes AA, Menten J, Phillips C, Fay M, Nishikawa R, Cairncross JG, Roa W, Osoba D, Rossiter JP, Sahgal A, Hirte H, Laigle-Donadey F, Franceschi E, Chinot O, Golfinopoulos V, Fariselli L, Wick A, Feuvret L, Back M, Tills M, Winch C, Baumert BG, Wick W, Ding K, Mason WP (2017) Short-Course Radiation plus Temozolomide in Elderly Patients with Glioblastoma. N Engl J Med 376: 1027-1037 doi:10.1056/NEJMoa1611977

29. Minniti G, De Sanctis V, Muni R, Rasio D, Lanzetta G, Bozzao A, Osti MF, Salvati M, Valeriani M, Cantore GP, Maurizi Enrici R (2009) Hypofractionated radiotherapy followed by adjuvant chemotherapy with temozolomide in elderly patients with glioblastoma. Journal of neuro-oncology 91: 95-100 doi:10.1007/s11060-008-9689-z 
30. Poon MTC, Sudlow CLM, Figueroa JD, Brennan PM (2020) Longer-term ( $\geq 2$ years) survival in patients with glioblastoma in population-based studies pre- and post-2005: a systematic review and meta-analysis. Scientific reports 10: 11622 doi:10.1038/s41598-020-68011-4

\section{Tables}




\section{Table 1. Patient and tumor characteristics in 33 patients diagnosed with butterfly glioblastoma between 01/01/2007 and 31/12/2014}

n $(\%)$

\section{Patient characteristics}

Female

$20(60.6 \%)$

Age $\geq 70$ years

$13(39.4 \%)$

Karnofsky Performance Status $<70$

$8(24.2 \%)$

Charlson comorbidity score $\geq 7$

$5 \quad(15.2 \%)$

Symptom(s) at time of diagnosis

$\begin{array}{lrl}\text { Cognitive impairment } & 23 & (69.7 \%) \\ \text { Headache } & 11 & (33.3 \%) \\ \text { Epilepsy } & 6 & (18.2 \%) \\ \text { Paresis } & 8 & (24.2 \%) \\ \text { Dizziness } & 7 & (21.2 \%) \\ \text { Central facial palsy } & 4 & (12.1 \%) \\ \text { Dysphasia } & 3 & (9.1 \%) \\ \text { Hemianopia } & 2 & (6.1 \%)\end{array}$

\section{MRI characteristics}

Main tumor location

Frontal $\quad 9$ (27.3\%)

$\begin{array}{lll}\text { Temporal } & 8 & (24.2 \%) \\ \text { Occipital } & 8 & (24.2 \%) \\ \text { Deep-seated } & 6 & (18.2 \%) \\ \text { Parietal } & 2 & (6.1 \%)\end{array}$

Corpus callosum affection ${ }^{\mathrm{a}}$

$\begin{array}{lrl}\text { Rostrum/genu } & 21(63.6 \%) \\ \text { Body } & 25 \quad(75.8 \%) \\ \text { Splenium } & 14 \quad(42.4 \%)\end{array}$

Tumor distribution

Left skewed

13 (39.4\%)

Right skewed

Symmetric

$7 \quad(21.2 \%)$

Tumor volumes

T1-weighted contrast-enhanced MRI $\left(\mathrm{cm}^{3}\right)$

$41 \quad(26-73)$

T2-weighted/FLAIR MRI $\left(\mathrm{cm}^{3}\right)$

137 (83-229)

Ratio T2/FLAIR:T1 volumes

$3.2(2.4-4.4)$

Hypothalamus involvement

$11 \quad(33.3 \%)$

Basal ganglia involvement

$14 \quad(42.4 \%)$

Blood vessel affection

$20 \quad(60.6 \%)$

Necrosis

$28 \quad(84.8 \%)$

Flow void

$23 \quad(69.7 \%)$

Mass effect $b$

$16 \quad(48.5 \%)$

Molecular characteristics $(n=11)^{C}$

MGMT promoter methylation status

Inconclusive 
Tumor volumes and T2/FLAIR:T1 ratio presented as median (IQR), all others as absolute numbers (\%). Tumor volumes defined as contrast enhancement and necrosis in T1-weighted MRI and tumor associated non-enhancing hyperintense lesions in T2-weighted/FLAIR MRI. a Sum exceeding 100\% due to the affection of multiple regions in several patients. ${ }^{b}$ Mass effect defined as midline shift $\geq 1 \mathrm{~mm}$. ${ }^{\mathrm{c}}$ Presented as absolute numbers and \% of patients with histological sample available for re-evaluation and molecular analyses. Abbreviations: MRI=Magnetic Resonance Imaging; FLAIR=fluid attenuated inversion recovery; $\mathrm{cm}^{3}=$ cubic centimetres; MGMT=O(6)-methylguanine-DNA methyltransferase. 
Table 2. Treatment and complications in 33 patients diagnosed with butterfly glioblastoma between 01/01/2007 and 31/12/2014.

n $(\%)$

\section{Primary treatment}

Surgery

None

$18(54.5 \%)$

Biopsy

$11(33.3 \%)$

Resection

$4 \quad(12.1 \%)$

Chemoradiotherapy

None

$6 \quad(18.2 \%)$

Short-course

$19 \quad(57.6 \%)$

Stupp protocol ${ }^{\mathrm{a}}$

8 (24.2\%)

Number of adjuvant TMZ courses

3 (1-9)

Number of treatment modalities ${ }^{b}$

None (best supportive care)

$6 \quad(18.2 \%)$

1 modality

$7 \quad(21.2 \%)$

2 modalities

$16 \quad(48.5 \%)$

3 modalities

$4 \quad(12.1 \%)$

Anti-tumor treatment last 30 days of life

$6 \quad(18.2 \%)$

\section{Complications during follow-up}

Surgical complications ${ }^{\mathrm{e}}$

$2(13.3 \%)$

Epileptic seizure

$13(39.4 \%)$

Venous thromboembolism

$10(30.3 \%)$

Hematotoxicity $^{\mathrm{f}}$

$3(15.8 \%)$

Osteoporosis

$4 \quad(12.1 \%)$

Treatment at recurrence $(n=18)^{C}$

Best supportive care

$11(61.1 \%)$

Surgical resection

$2(11.1 \%)$

Stereotactic radiosurgery

$1 \quad(5.6 \%)$

Re-irradiation

$0 \quad(0.0 \%)$

TMZ monotherapy

$5(27.8 \%)$

CCNU-based chemotherapy $\left(\mathrm{PCV}^{\mathrm{d}}\right)$

$1 \quad(5.6 \%)$

Bevacizumab and nitrosoureas (CCNU or BCNU)

$2(11.1 \%)$

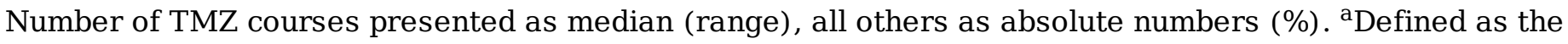
completion of radiation therapy (60 Gy in 2 Gy fractions) with concurrent TMZ and at least one of six planned

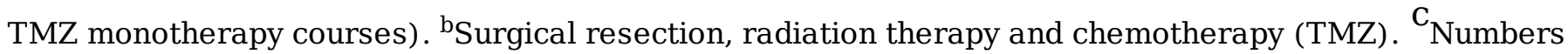
include 18 patients where relapse was detected, while two patients with no sign of recurrence during follow-up and 13 patients who had a continuous deterioration and died without being diagnosed with recurrence were not included. ${ }^{\mathrm{d}}$ Procarbazine, Lomustine (CCNU), and Vincristine. ${ }^{e}$ Complications among 15 patients who had undergone biopsy or resection included cerebral infarction and paresis $(n=1)$ and increasing dysphasia $(n=1)$. ${ }^{\mathrm{f}} \mathrm{CTCAE} \geq$ grade 3 among 19 patients receiving TMZ. Abbreviations: CTCAE=Common Terminology Criteria 
for Adverse Events; TMZ=Temozolomide; CCNU=cyclonexyl-chloroethyl-nitrosourea (Lomustine); $\mathrm{BCNU}=\mathrm{Bis}$ (Chloroethyl) nitrosourea (Carmustine). 
Table 3. Unadjusted and adjusted analyses on overall survival in 33 patients diagnosed with butterfly glioblastoma between 01/01/2007 and 31/12/2014.

\begin{tabular}{|c|c|c|c|c|}
\hline \multirow[b]{2}{*}{ Variable } & \multicolumn{2}{|l|}{ Unadjusted } & \multicolumn{2}{|c|}{ Adjusted for sex, age, and KPS } \\
\hline & $\mathrm{HR}(95 \% \mathrm{CI})$ & $\mathrm{P}$ value & HR $(95 \% \mathrm{CI})$ & $\mathrm{P}$ value \\
\hline \multicolumn{5}{|l|}{ Sex } \\
\hline Male & Ref. & & Ref. & \\
\hline Female & $0.74(0.36-1.52)$ & 0.41 & $0.74(0.36-1.54)$ & 0.42 \\
\hline Age (per year) & $1.06(1.03-1.10)$ & 0.001 & $1.06(1.02-1.10)$ & 0.003 \\
\hline \multicolumn{5}{|l|}{ KPS } \\
\hline$\geq 70$ & Ref. & & Ref. & \\
\hline$<70$ & $2.96(1.21-7.21)$ & 0.02 & $1.86(0.73-4.74)$ & 0.19 \\
\hline \multicolumn{5}{|l|}{ Tumor side } \\
\hline Left & Ref. & & Ref. & \\
\hline Right & $1.28(0.57-2.86)$ & 0.56 & $1.51(0.63-3.59)$ & 0.35 \\
\hline Equal & $1.95(0.75-5.05)$ & 0.17 & $1.77(0.64-4.91)$ & 0.27 \\
\hline \multicolumn{5}{|l|}{ Main tumor location } \\
\hline Frontal lobe & Ref. & & Ref. & \\
\hline Temporal lobe & $3.59(1.11-11.65)$ & 0.03 & $1.32(0.31-5.70)$ & 0.71 \\
\hline Occipital lobe & $1.59(0.56-4.52)$ & 0.39 & $1.70(0.58-4.98)$ & 0.33 \\
\hline Parietal lobe & $0.43(0.05-3.43)$ & 0.42 & $0.99(0.11-9.03)$ & 1.00 \\
\hline Deep-seated & $6.69(1.84-24.35)$ & 0.004 & $4.58(1.15-18.20)$ & 0.03 \\
\hline MRI T1 volume $\left(\right.$ per $\left.\mathrm{cm}^{3}\right)$ & $1.00(0.99-1.01)$ & 0.68 & $1.00(0.99-1.02)$ & 0.56 \\
\hline MRI T2/FLAIR volume $\left(\right.$ per $\left.\mathrm{cm}^{3}\right)$ & $1.00(1.00-1.01)$ & 0.33 & $1.00(1.00-1.01)$ & 0.15 \\
\hline T2/FLAIR:T1 ratio (per unit) & $1.01(0.88-1.16)$ & 0.90 & $0.95(0.79-1.14)$ & 0.58 \\
\hline Hypothalamus involvement & $2.02(0.95-4.32)$ & 0.07 & $1.36(0.63-2.93)$ & 0.44 \\
\hline Basal ganglia involvement & $1.63(0.79-3.35)$ & 0.19 & $1.23(0.54-2.83)$ & 0.62 \\
\hline Blood vessel involvement & $0.57(0.27-1.19)$ & 0.13 & $0.94(0.40-2.23)$ & 0.89 \\
\hline Mass effect (per mm midline shift) & $0.95(0.87-1.04)$ & 0.24 & $0.98(0.89-1.08)$ & 0.67 \\
\hline Necrosis & $0.64(0.24-1.71)$ & 0.38 & $0.37(0.13-1.06)$ & 0.06 \\
\hline Flow void & $0.64(0.29-1.41)$ & 0.27 & $0.97(0.40-2.35)$ & 0.95 \\
\hline
\end{tabular}

MGMT promoter methylation status

Unmethylated

Ref.

Methylated

$0.29(0.04-2.42) \quad 0.25$

$0.06(0.00-3.33)$

0.17

Primary treatment

Two or three modalities

Ref.

One modality

$2.37(0.95-5.96) \quad 0.07$

Ref.

Best supportive care

$8.10(2.65-24.79) \quad<0.001$

1.67 (0.43-6.51)

0.46

$5.11(1.09-23.89)$

0.04

HR, 95\% CI and p values calculated by unadjusted and adjusted Cox proportional hazards regression. Deepseated location defined as tumor mainly located in thalamus, basal ganglia, capsula interna, splenium corpus callosum, or mesencephalon. Tumor volumes in cubic centimetres $\left(\mathrm{cm}^{3}\right)$. Mass effect measured as midline shift in millimetres $(\mathrm{mm})$. Volumes defined as contrast-enhancing tumor volume in T1-weighted MRI and tumor associated non-enhancing hyperintense lesion in T2-weighted/FLAIR MRI. Only patients with conclusive result were included in MGMT analyses $(n=9)$. Treatment modalities included surgical resection, radiation therapy, and Temozolomide concurrent and/or adjuvant. $\mathrm{P}$ values $<0.05$ were considered statistically significant and marked in bold. $\mathrm{HR}=$ hazard ratio; $\mathrm{CI}=$ confidence interval; KPS=Karnofsky Performance Status; MRI=magnetic resonance imaging; FLAIR=fluid attenuated inversion recovery; $\mathrm{MGMT}=\mathrm{O}$ (6)methylguanine-DNA methyltransferase. 


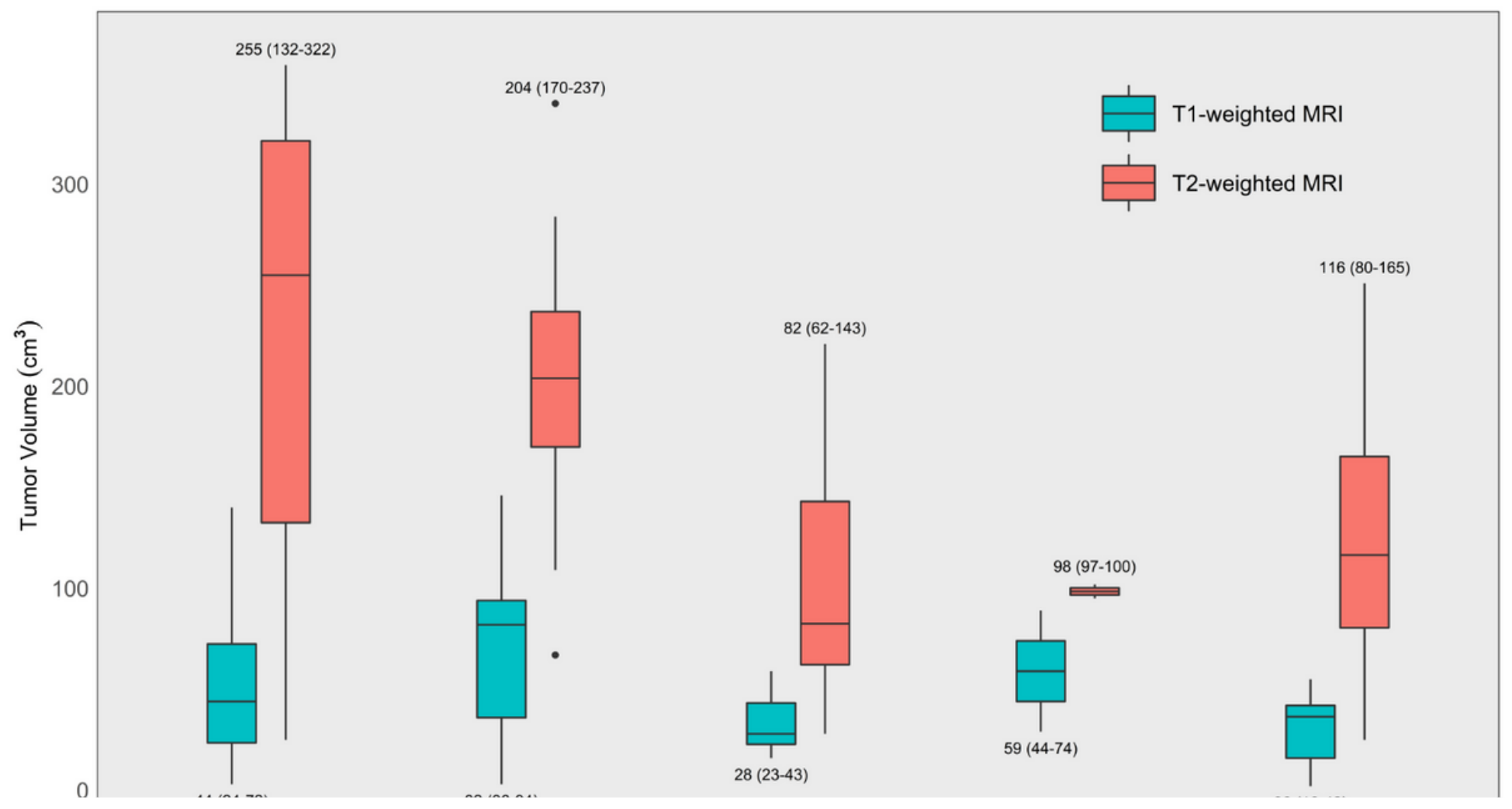

Figure 1

Median (Interquartile range (IQR)) contrast-enhancing tumor volume in T1-weighted Magnetic Resonance Imaging (MRI) and median (IQR) volume of tumor associated non-enhancing hyperintense lesions in T2weighted and/or fluid attenuated inversion recovery (FLAIR) MRI according to tumor location in 33 patients diagnosed with butterfly glioblastoma between 01/01/2007 and 31/12/2014 

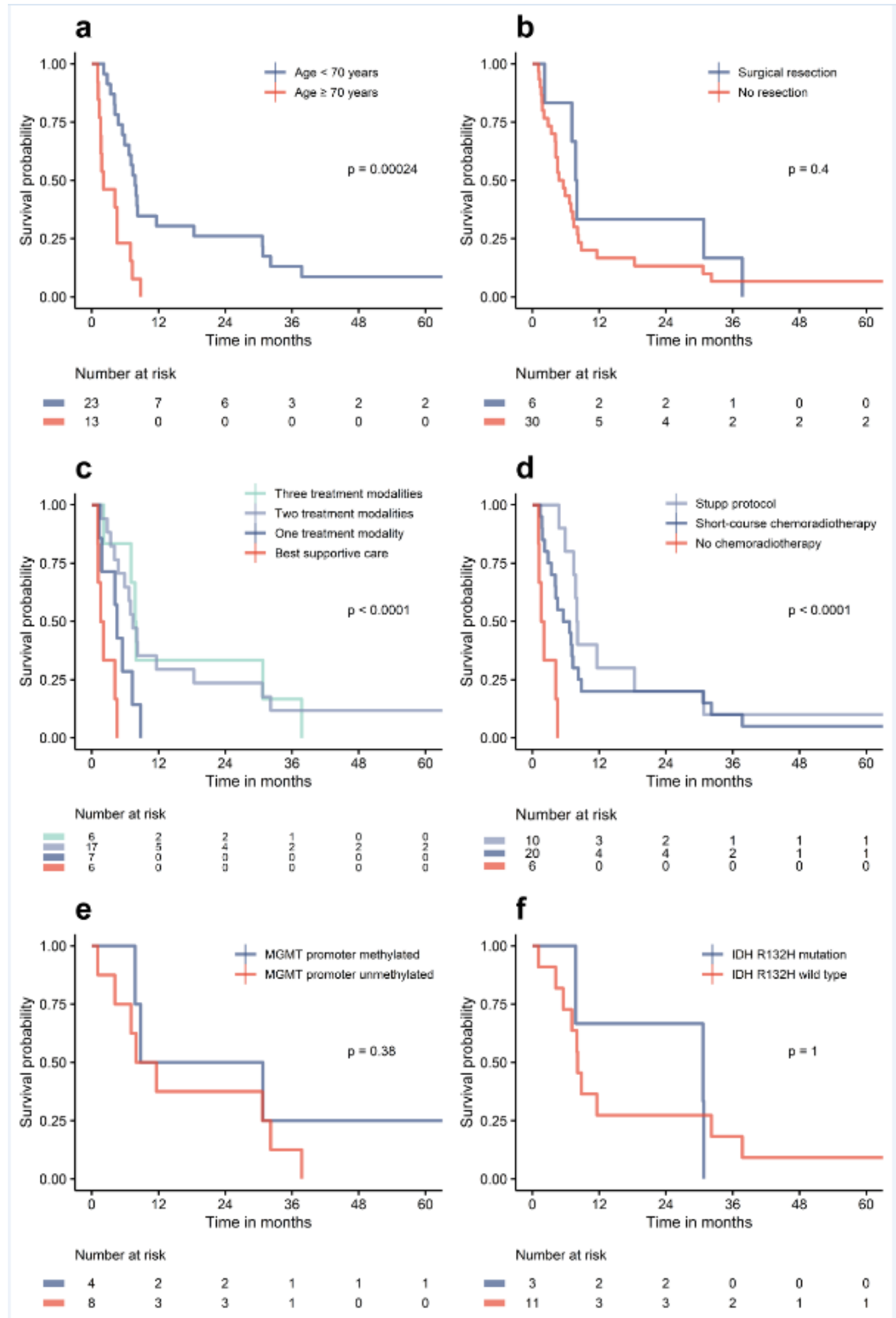

\section{Figure 2}

Kaplan-Meier curves of survival probability in 33 patients diagnosed with butterfly glioblastoma between 01/01/2007 and 31/12/2014. Cumulative survival in months. Survival by a) age, b) surgical resection, c) chemoradiotherapy regimen, and d) number of treatment modalities. Comparison of groups by log rank test. $P$ values $<0.05$ were considered statistical significant 